\title{
HIGH RESOLUTION X-RAY CHARACTERIZATION OF PERIODICALLY DOMAIN-INVERTED NONLINEAR OPTICAL CRYSTALS
}

\author{
Z.W. $\mathrm{Hu}^{*}$ \\ Department of Physics, University of Warwick, Coventry, CV4 7AL, UK \\ Clarendon Laboratory, University of Oxford, Parks Road, Oxford, OX1 3PU, UK \\ P.A. Thomas \\ Department of Physics, University of Warwick, Coventry, CV4 7AL, UK \\ J. Webjörn
}

Optoelectric Research Centre, University of Southampton, Southampton SO9 5NH, UK

\begin{abstract}
A high-resolution triple-axis diffractometer has been used for the structural characterization of periodically domain-inverted nonlinear optical crystals of $\mathrm{KTiOPO}_{4}$ and $\mathrm{LiNbO}_{3}$. Striations have been revealed in high strain-sensitivity multiple-crystal topographs of the domain-inverted regions of both these samples and these are dominated by orientation contrast. The combination of high-resolution reciprocal-space mapping and topography has shown that the extended diffraction streak in the $q_{[2 \overline{0}]}$ direction for domain-inverted $\mathrm{LiNbO}_{3}$ originates from the "minutely misoriented structure" which is related to the original configuration of dislocations. The reason for the generation of the structural imperfections via the domain-inversion processing is interpreted in terms of the converse piezoelectric effect.
\end{abstract}

*On leave from National Laboratory of Solid State Microstructures, Nanjing University, Nanjing, China 


\section{Introduction}

Quasi-phase matched second-harmonic generation based on periodically domaininverted structures has received great attention for novel applications beacuse of the high nonlinear conversion efficiency that can be ublained and the possibility of phasematching arbitrary wavelengths. Techniques to fabricate periodic domain inversion in $\mathrm{LiNbO}_{3}(\mathrm{LN})$ [2-5], $\mathrm{LiTaO}_{3}(\mathrm{LT})[6]$, and $\mathrm{KTiOPO}_{4}(\mathrm{KTP})[7,8]$, such as proton or ion exchange, electric field poling and electron lithography, have been developed rapidly. However, so far it is clear that the detailed effects of the periodic-poling techniques on the crystals and the physical mechanisms of these methods have not been well understood. This lack of fundamental understanding will adversely affect the development of reliable and reproducible periodically-poled devices in the future. Therefore, the establishment of a structural and microstructural understanding of periodic poling is of considerable importance and interest.

High-resolution $X$-ray diffraction and topography have been extensively used for the characterization of semiconductor thin films in recent years because the techniques are both highly strain-sensitive and non-destructive[9-12]. To our knowledge, these techniques have not been applied thus far to the structural inspection of periodically domain-inverted crystals. In the present paper, multiple-crystal multiple-reflection $X$ ray topography combined with high-resolution reciprocal-space mapping is employed for the structural characterization of periodically domain-inverted KTP and LN crystals. The wealth of structural information revealed in the early experiments suggests a promising new application of high-resolution $X$-ray diffraction in the characterization of nonlinear optical materials.

\section{Experimental Procedures}

A $20 \mu \mathrm{m}$ periodic domain-inverted pattern in an optical-grade hydrothermally-grown KTP crystal was written by scanning an electron beam on the (00i) (or $C^{-}$) face[8]. The sample was then etched using a 2:1 molar mixture of $\mathrm{KOH}$ and $\mathrm{KNO}_{3}$. The chemical etching showed that the domain-reversal had penetrated the full thickness of the 
crystal. Fig. 1 shows an optical transmission micrograph of the periodically domaininverted structure on the etched $C^{-}$face of KTP. The period of the domain inversion is observed to be $20 \mu \mathrm{m}$.

Periodic domain-inversion in a Z-cut LN sample was achieved by electric-field poling. The domain reversal occurred throughout the $1 \mathrm{~mm}$ thickness of the sample. The domain-inverted pattern on the $\mathrm{C}^{+}$face was revealed by etching in a solution of two parts $\mathrm{HNO}_{3}$ and one part $\mathrm{HF}$ and showed minus domains $5-6-\mu \mathrm{m}$ in width alternating with plus domains of $14-15 \mu \mathrm{m}$ in width (Fig.2). The LN sample was annealed for 4 days at $120^{\circ} \mathrm{C}$ before $\mathrm{X}$-ray diffraction measurements to relieve excess strain induced during the poling procedures.

A Philips high-resolution multiple-crystal multiple-reflection X-ray diffractometer (Fig.3)[13], which combines the advantages of a two-crystal four-reflection Ge 220 monochromator and $\mathrm{Ge} 220$ analyser, was then used both to take $\mathrm{X}$-ray topographs and to map the diffraction intensity in reciprocal space, using $\mathrm{Cu} \mathrm{K}_{\alpha 1}$ radiation. The monochromator used in the diffractometer consists of two U-shaped crystals each having its own $(n,-n)$ double-reflection geometry. This gives an incident $X$-ray beam of $12 "$ arc ( the intrinsic width of the Ge 220 reflection) or less in angular divergence. The dispersion effect arising from wavelength variation is negligible for this optical configuration. The $00 \overline{4}$ and $00 \overline{8}$ symmetric reflections were used for taking topographs of KTP, and the 006 symmetric reflection was used for LN. The penetration depth of $\mathrm{Cu}$ $\mathrm{K}_{\alpha 1}$ radiation into both these materials primarily depends on the $\mathrm{X}$-ray extinction distance[14], which is not less than $10 \mu \mathrm{m}$ for the reflections used. The horizontal resolution, which is determined by the crystal optics, is of the order of microns or less. The vertical resolution (i.e. the vertical divergence), which is given by the ratio of (sample-to-film $\times$ source height) to (source-to-sample distance), is lower than $150 \mu \mathrm{m}$ for the present case. In our study, the samples were usually oriented such that the domain walls were perpendicular to the plane of incidence of the $\mathrm{X}$-rays. The $\mathrm{X}$-ray diffraction topographs were recorded on $25 \mu \mathrm{m}$ ILFORD L5 nuclear-emulsion plates. 


\section{Experimental Results}

\section{III.I. Multiple Crystal Topography of KTP}

$00 \overline{4}$ and $00 \overline{8}$ symmetric reflections with a three-crystal five-reflection (3C5R) geometry were used for high strain-sensitivity $\mathrm{X}$-ray diffraction topography. The rocking curve widths of $00 \overline{4}$ and $00 \overline{8}$ reflections from the sample are 45" and 30" arc, respectively. They are larger than the angular and spectral divergence of the incident beam produced by the monochromator. The defect images occur as a result of deviation from the Bragg diffraction condition due to either a change of orientation $\theta$ or of lattice spacing $d$, or of both simultaneously. If the sample is oriented to correspond to the flank of the rocking curve, then the image contrast can be described in a simple manner[ 15$]$ and is given by

$$
\triangle \mathrm{I}=\mathrm{K}\left(\stackrel{\triangle \mathrm{d}}{\mathrm{d}} \tan \theta_{\mathrm{B}}+\delta \theta\right)
$$

where $\theta_{B}$ is the Bragg angle, $K$ is the slope of the rocking curve flanks, $\Delta d / d$ the local relative change in spacing of the diffracting planes, and $\delta \theta$ the component of the local lattice rotation projected into the incident plane. The lattice dilation $\Delta \mathrm{d} / \mathrm{d}$ and the lattice tilt $\delta \theta$ can be separated by high-resolution reciprocal-space mapping in two dimensions.

A demonstration of the difference in contrast for a domain-inverted region and an untreated area (i.e. not subjected to electron-beam writing) is given in Figs.4(a)-(e). These topographs were taken at the linear part of the rocking curve flanks. Fig.4(a) shows an $00 \overline{8}$ reflection topograph of the untreated region which is characterized by a relatively smooth distribution of intensity. However, obvious fluctuations in the contrast are revealed in $00 \overline{4}$ and $00 \overline{8}$ topograhs of the domain-inverted region(Fig.4(b) and (c)). The left-hand side of each of these two topographs corresponds to the untreated zone and the right-hand side is the domain-inverted region. Sets of vertical striations can be seen in the domain-inverted area. These striations appear essentially the same in the $00 \overline{4}$ and $00 \overline{8}$ topographs, but the sensitivity in Fig.4(c) is higher than that in Fig.4(b). 
The enhancement of the image contrast at the boundary of the domain-inverted region in Fig.4(b) can be attributed mainly to horizontal condensation of the image by the contraction factor $\sin \theta_{B}$ for a symmetric reflection. The angles of incidence for the 004 and 008 reflections are about 14.17 and 33.05 degrees, respectively. The steep slopes of the rocking curve flanks for the $00 \overline{4}$ and $00 \overline{8}$ reflections are $1.4 \times 10^{6}$ and $2.3 \times 10^{6}$ (cps/ degree), respectively. This means that it is possible to detect lattice dilations as low as $10^{-7}$ or lattice rotations as small as $10^{-3}$ second of arc on the $X$-ray topographs. The striation contrast disappeared when X-ray topographs were taken in the relatively flat regions of the rocking curve flanks. A two-dimensional reciprocal-space map of the $00 \overline{8}$ reciprocal lattice point shows that the distortions generated within the domain-inverted regions mainly result from the lattice tilt $\delta \theta$. However, this map was also affected by the presence of some small cracks in the crystal produced by the etching process. The difuse scattering close to the Bragg peak is essentially isotropic and not directional as obtained from the LN sample (Fig.7), the diffraction space map was only used as a guide for the 3C5R topographs and was not analysed further.

Careful inspection of the images shows that the spacings of the striations revealed in the $\mathrm{X}$-ray topographs are larger than the period of the domain inversion. This indicates that the striations are not directly associated with the domain walls themselves. An interpretation of the physical origin of these features is given in section IV.

\section{III.II Multiple-Crystal Topography and High-Resolution Diffraction Mapping of LN}

The 006 symmetric reflection with 3C5R geometry was used for rocking curves and high-resolution topography. Two diffraction profiles from the untreated and domaininverted regions are shown in Fig.5. It is seen that the full width at half maximum (FWHM) is broadened slightly subsequent to the domain-inversion processing from 19" (untreated region) to $22^{\prime \prime}$ arc (domain-inverted region). The defect contrast with the probe positioned on the rocking curve flanks can be understood from Eq.(1). X-ray topographic observations (Fig.6) indicate that a change in the defect contrast has arisen 
in the domain-inverted region compared with that in the untreated region which is dominated by orientation contrast. Sets of vertical striations, which are similar to those observed in KTP, are shown in Fig.6(b).

The principle and advantages of high-resolution reciprocal-space mapping using the high-resolution triple-axis diffractometer have been described by Fewster[16]. Compared with a rocking curve (a one-dimensional scan in reciprocal-space), twodimensional reciprocal-space mapping is more effective in revealing structural information. Two representative 006 reciprocal-space maps are shown in Fig.7(a) \& (b). The former corresponds to the untreated region whereas the latter comes from the domain-inverted zone. A much extended diffraction streak in the $\omega$-scan direction (i.e., along $\left.q_{[2 i 0]}\right)$ appears in the diffraction map for the domain-inverted region(Fig.7(b)). It should be noted that the elongated diffuse-scattering streak always emerges on the diffraction space map whenever the domain walls are perpendicular to the plane of incidence at all points in the inverted regions. Therefore, it seems that this diffraction streak along the $q_{[2 \bar{i} 0]}$ direction is truly a consequence of the periodic domain-inversion process.

A series of high-resolution four-crystal seven-reflection (4C7R) topographs was taken to image specific regions of the diffraction-space maps. Fig.8(a) is an X-ray topograph of the diffuse-scattering obtained from the untreated area of the sample with $\Delta \omega=28$ " from the Bragg peak in Fig.7(a). The strain fields are clearly revealed with dislocation images lying in the [120] direction. Evidently, the dominant contribution to the diffuse-scattering in the untreated region comes from these dislocations. Fig. 8(b) is an X-ray diffuse-scattering image of the domain-inverted region with $\Delta \omega=27.9^{\prime \prime}$ from the Bragg peak in Fig.7(b). Interestingly, misoriented "blocks" in Fig.8(b) are clearly revealed rather than sets of vertical dislocations as in Fig.8(a). These blocks appear as regions of slightly increased misorientation which changes rather smoothly from block to block without the presence of sharply defined grain boundaries. These can be considered as "minutely misoriented structure" rather than mosaic structure since the 
latter term conventionally implies the presence of low-angle grain boundaries. It can be seen from both the mapping and the topography that the striations revealed in the domain-inverted regions (Fig.6(b)) are relevant to the misoriented structure and are dominated by orientation contrast. We have examined some other regions near the boundary between the domain-inverted and untreated regions using $X$-ray diffusescattering. The defect structures revealed are the same as those described above - these are therefore representative of the sample as a whole. The extended diffuse-scattering in the $\mathbf{q}_{[2 \overline{1} 0]}$ direction is caused mainly by this "minutely misoriented structure" along the [120] direction which is generated in the domain-inverted region by the poling process. Note that the misoriented contrast and the elongated diffuse scattering will disappear if the incidence plane is parallel to the domain walls because this misoriented structure is associated specifically with the [120] direction. The topographs (Fig.8) show that the spatial distribution of the misoriented structure follows that of the original dislocations, suggesting that its formation is intimately correlated with the initial ditribution of dislocations in the untreated LN crystal.

\section{Discussions}

High-resolution X-ray topography and mapping of periodically domain-inverted $L N$ and KTP shows that extra defect structures have been generated in addition to the periodic domain inversion. This is suggested to be a general consequence of the domain-inversion processing using both the electron-beam lithography and the electricfield poling techniques. Because of the converse piezoelectric effect, the samples are strained when subjected to electron bombardment or applied electric field. During the poling treatments, the applied electric fields $\left(\mathrm{E}_{3}\right)$ are in the [001] direction. For KTP, the components of the induced strain are

$$
\begin{aligned}
& S_{11}=d_{311} E_{3} \\
& S_{22}=d_{322} E_{3} \\
& S_{33}=d_{333} E_{3}
\end{aligned}
$$


For $\mathrm{LN}$, the components of the induced strain are

$$
\begin{aligned}
& S_{11}=d_{311} E_{3} \\
& S_{22}=d_{311} E_{3} \\
& S_{33}=d_{333} E_{3}
\end{aligned}
$$

Therefore, the electric field created because of the electron-beam bombardment for KTP or the applied electric field for $\mathrm{LN}$, cause three mutually perpendicular components of the strain in the treated regions of the sample as well as stimulating domain-inversion. In principle, the strain-induced and strain-free areas should have the same periodicity as the applied poling field. However, in fact, it appears that inhomogeneous and complicated strain fields are generated within the samples because of the interaction between the newly induced strains and the initial strain fields present in the samples. The induced strains are relaxed to some extent on removal of the applied electric field and in the subsequent heat treatments. We suggest that these strains are relaxed by the generation of local lattice tilts and that these are the major source of the striations observed in topographs of the domain-inverted regions in KTP and LN. In relaxing the induced strains, increased misorientation occurs more easily around existing defects than in near-perfect areas because a certain degree of tilting already pre-exists in the imperfect regions. This can be inferred from the fact that the misoriented structure generated is quite similar to the initial distribution of the dislocations in the substrate(Fig.8).

In general, the generation of defects through the domain-inversion process occurs in the same manner for KTP as for LN. But this effect seems amplified in LN compared with that in KTP possibily because LN is harder to pole with electric field than KTP [8] meaning that it has been subjected to a larger electric field. The order of magnitude of the induced strains is determined by both the applied field and the piezoelectric moduli of the sample measured. The piezoelectric moduli of $L N, d_{311}$ and $d_{333}$, are $-0.86 \times 10^{-12}$ $C / N$ and $16.2 \times 10^{-12} \mathrm{C} / \mathrm{N}$, respectively. The applied electric field $(E)$ is $20 \mathrm{KV} / \mathrm{cm}$. So, 
$S_{11}=S_{22}=-1.72 \times 10^{-6}, S_{33}=3.24 \times 10^{-5}$. The induced strains are therefore large enough to be detected by high-resolution X-ray diffraction. Unfortunately, we are unable to present the same data for KTP because measured values for the piezoelectric coefficients are not available. But we can reasonably expect that the induced strains in KTP are lower than those in LN because the poling in KTP is a lower energy process [8,17-18]. So far, we have concentrated experimentally only on the 006 reflection from planes parallel to the domain-inverted surface because of its large structure factor. This reflection is sensitive to the component of the strain normal to the surface $\left(S_{33}\right)$, which is the largest of the induced strain components. In the periodic-poling experiments, the magnitude of the applied electric field must be carefully chosen because too high a field could generate a mosaic structure which could be detrimental to the nonlinear optical properties of the crystals because of scattering losses.

\section{Conclusions}

We have applied high-resolution diffraction and topography to the characterization of periodically domain-inverted KTP and LN crystals. It has been shown that an undesirable generation of defects occurs in addition to the desired domain-inversion process. The formation of striations termed a "minutely misoriented structure" in these domain-inverted crystals can be interpreted via the converse piezoelectric effect and the application of a spatially periodic electric field in these experiments.

The wealth of structural information revealed so far in these experiments provides a clear demonstration of the great potential of high-resolution $\mathrm{X}$-ray diffraction in the study of nonlinear optical crystal devices. Further work aimed at quantitative analysis of the diffraction information herein is currently in progress.

ZWH would like to acknowledge the financial support of SERC under grant number GR/T 02414. Also, we are grateful to the referees for their most stimulating criticisms of the first version of our manuscript. 


\section{References}

1. J.A. Armstrong, N. Bloembergen, J. Ducuing, and P.S. Pershan, Phys. Rev. 127, 1918(1962)

2. D. Feng, N.B. Ming, J.F. Hong, Y.S. Yang, J.S. Zhu, Z. Yang, and YN. Wang, Appl. Phys. Lett.37,607(1980)

3. Nai-Ben Ming, Jing-Feng, Hong, and Duan Feng, J. Mater. Sci.17, 1663(1982)

4. M.M. Fejer, G.A. Magel, and EJ. Lim, Proc. SPIE 1148, 213(1989)

5. M Yamada, N. Nada, M. Saitoh, and K. Watanable, Appl. Phys. Lett.62, 435(1993)

6. K. Mizuuchi, K. Yamamoto, and T. Taniuchi, Appl. Phys. Lett.59, 1538(1991)

7. CJ.v.d. Poel, J.D. Bierlein, J.B. Bowen, and S. Colak, Appl. Phys. Lett. 57, 2074(1990)

8. M.C. Gupta, W.P. Risk, A.C,G. Nutt, and S.D. Lau, Appl. Phys. Lett.63, 1167(1993)

9. D.K. Bowen, in Application of Synchrotron Radiation (New York, Gordon and Breach, 1989), P.91

10. B.K. Tanner, in Analysis of Microelectronic Materials and Devices(New York, Wiley, 1991), P.609

11. P.F. Fewster, Applied Surface Science 9, 50(1991)

12. R. Köhler, Appl. Phys. A58, 149(1994)

13. P.F. Fewster, J. Appl. Cryst.22, 64(1989)

14. R.W. Armstrong, in Characterization of Crystal Growth Defects by X-ray Methods (New York, Plenum Press, 1980), p.353

15. U. Bonse, in Direct Observation of Imperfections in Crystal (New York, Interscience, 1961), P. 431

16. P.F. Fewster, Appl. Phys. A58, 121(1994)

17. J.D. Bierlein, and H. Vanherrzeele, J. Opt. Soc. Am B6, 622(1989)

18. Z.W. Hu, P.A. Thomas, Mool C. Gupta, and W.P. Risk, unpublished 
Fig.1 Optical micrograph showing periodically domain-inverted pattern on the $\mathrm{C}^{-}$face of a $\mathrm{KTiOPO}_{4}$ crystal. The minus-domains and plus-domains emerge alternately, with domain walls (darker lines) parallel to the [010] direction.

Fig. 2 Optical micrograph showing periodically domain-inverted structure on the $\mathrm{C}^{+}$ face of a $\mathrm{LiNbO}_{3}$, with minus-domains 5-6- $\mu \mathrm{m}$ in width alternating with plus-domains of $14-15-\mu \mathrm{m}$ in width. The domain walls (darker lines) are perpendicular to the [100] direction.

Fig.3 High-resolution four-crystal seven-reflection diffractometer geometry. The sample axis of rotation, $\omega$, and that of the detector and analyser crystal, $\omega^{\prime}$, are coaxial about the sample face. The three-crystal five-reflection arrangement can be obtained by rotating the detector about $\omega^{\prime}$ to receive the diffracted beam from the sample.

Fig. 4. Three-crystal five-reflection topographs of a periodically domain-inverted $\mathrm{KTiOPO}_{4}$ crystal. The horizontal direction is the a-axis. (a) the untreated region, $00 \overline{8}$ symmetric reflection; (b) striations shown within the domain-inverted region(right-hand side), $00 \overline{4}$ symmetric reflection; (c) striations shown within the domain-inverted region(right-hand side), $00 \overline{8}$ symmetric reflection. The magnification of the images is marked in Fig. 4 (c).

Fig.5. Rocking curves of the 006 reflection for the untreated (solid line) and domaininverted (dashed line) LN.

Fig.6. 3C5R high-resolution topographs of LN. The horizontal direction is the a-axis. (a) the untreated region; (b) the domain-inverted region. Sets of vertical striations 
appear in Fig. 6 (b) compared with the defect images in the untreated region.

Fig.7. The 006 reciprocal-space maps for (a) the untreated region and (b) the domaininverted regions of $L N$. The $X$-axis and $Y$-axis, respectively, represent the $q_{[210]}$ and $\mathbf{q}_{[001]}$ directions. The extended diffraction streak along the $q_{[2 \overline{1} 0]}$ direction appears in Fig.7(b).

Fig.8. Weak-beam X-ray topographs of $L N$ taken at positions with (a) $\Delta \omega=28^{\prime \prime}$ and (b) $\Delta \omega=27.9^{\prime \prime}$, respectively, from the Bragg peaks in Fig.7(a) and (b). The horizontal direction is the a-axis. The vertical resolution is less than $150 \mu \mathrm{m}$, which causes the elongated vertical images of the dislocations. The magnification of the images is marked in Fig.7(a). 




$$
\text { Fig. } 1
$$

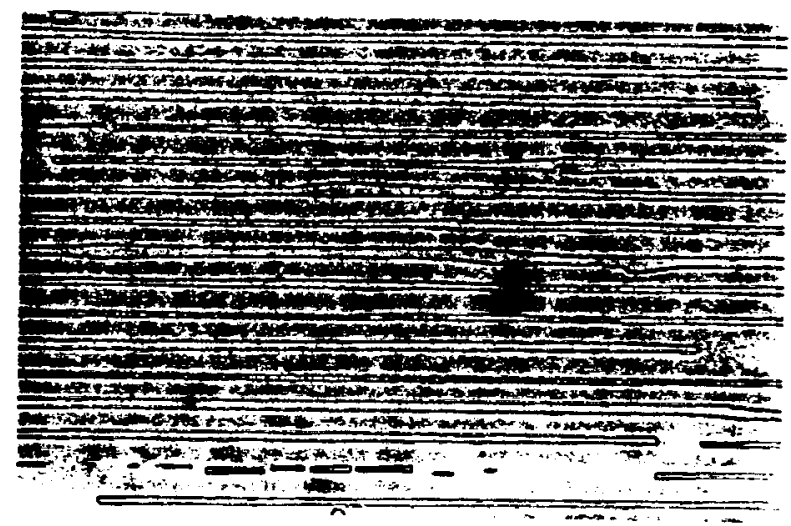

$$
\text { Fig. } 2
$$




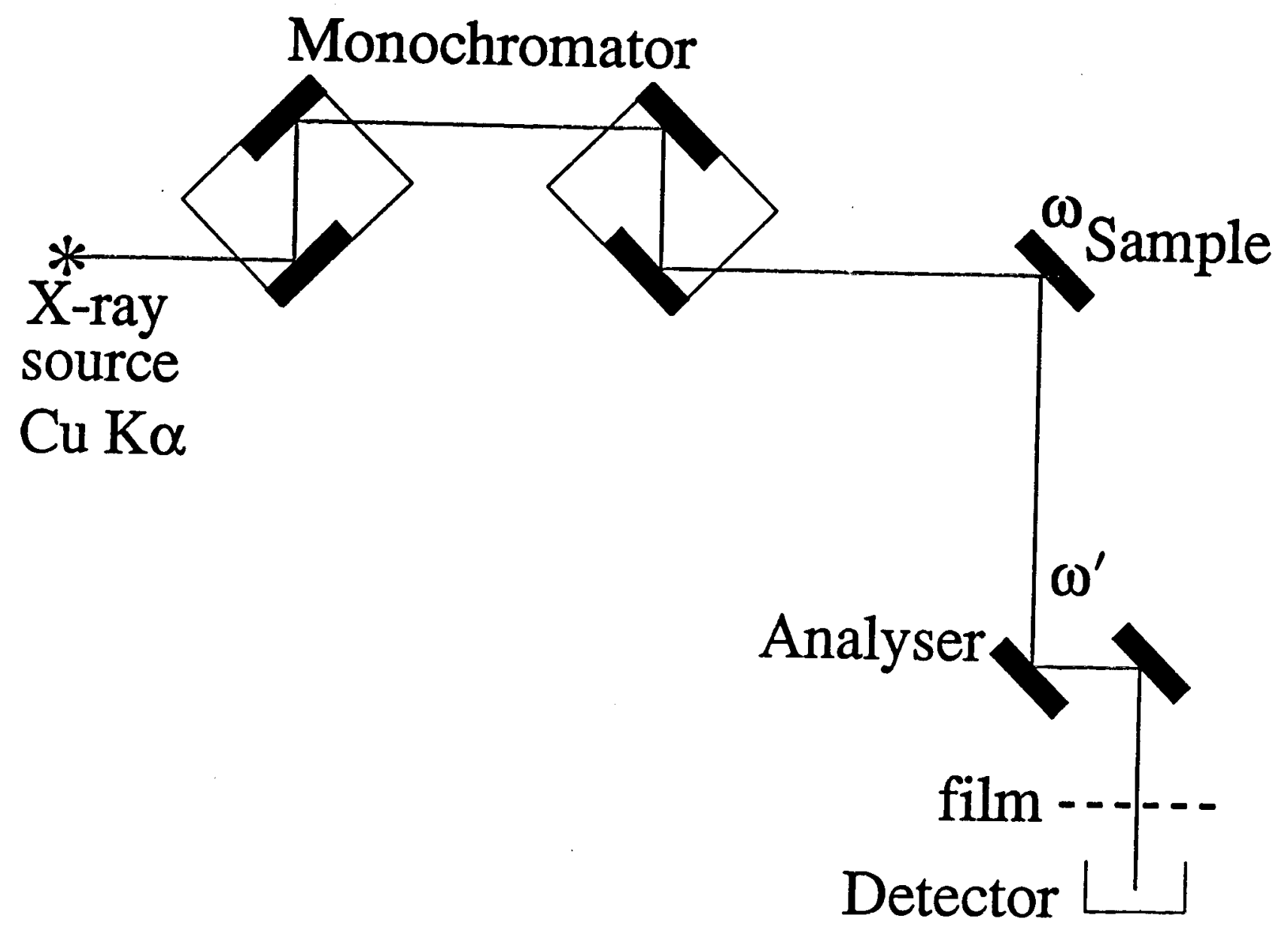

Fid 3 

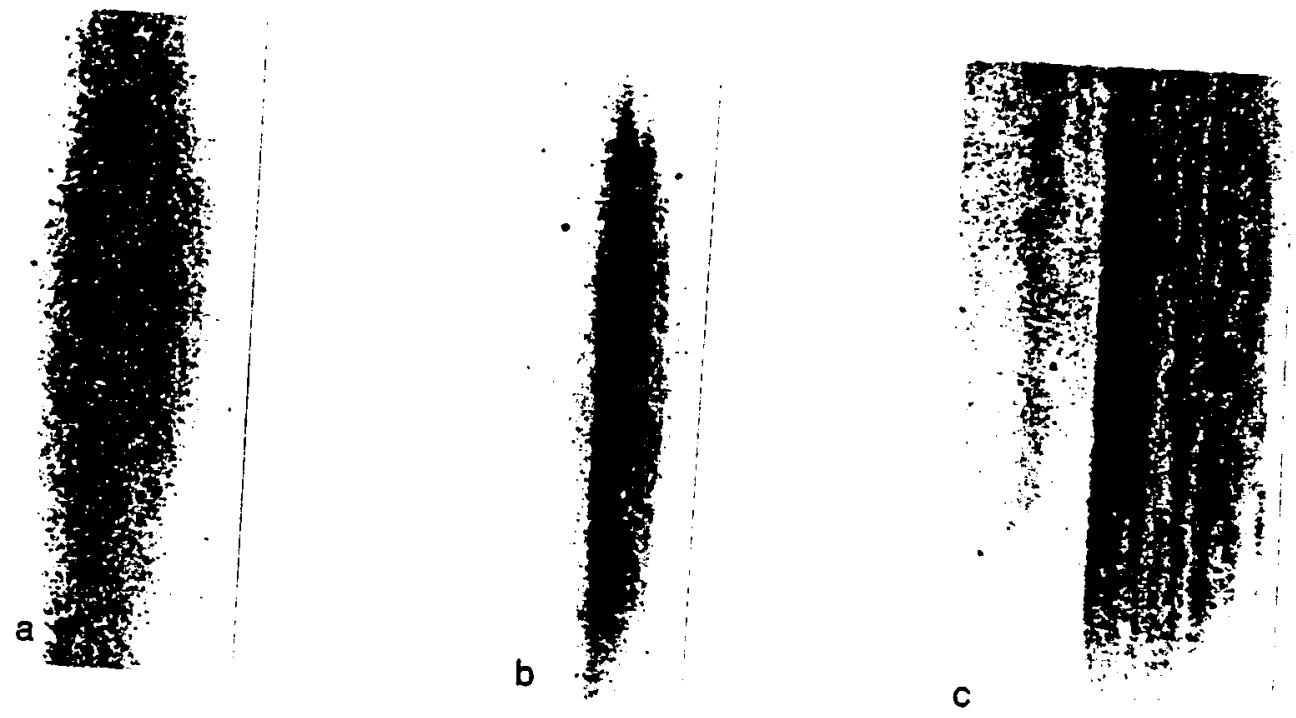

Fig. 4 


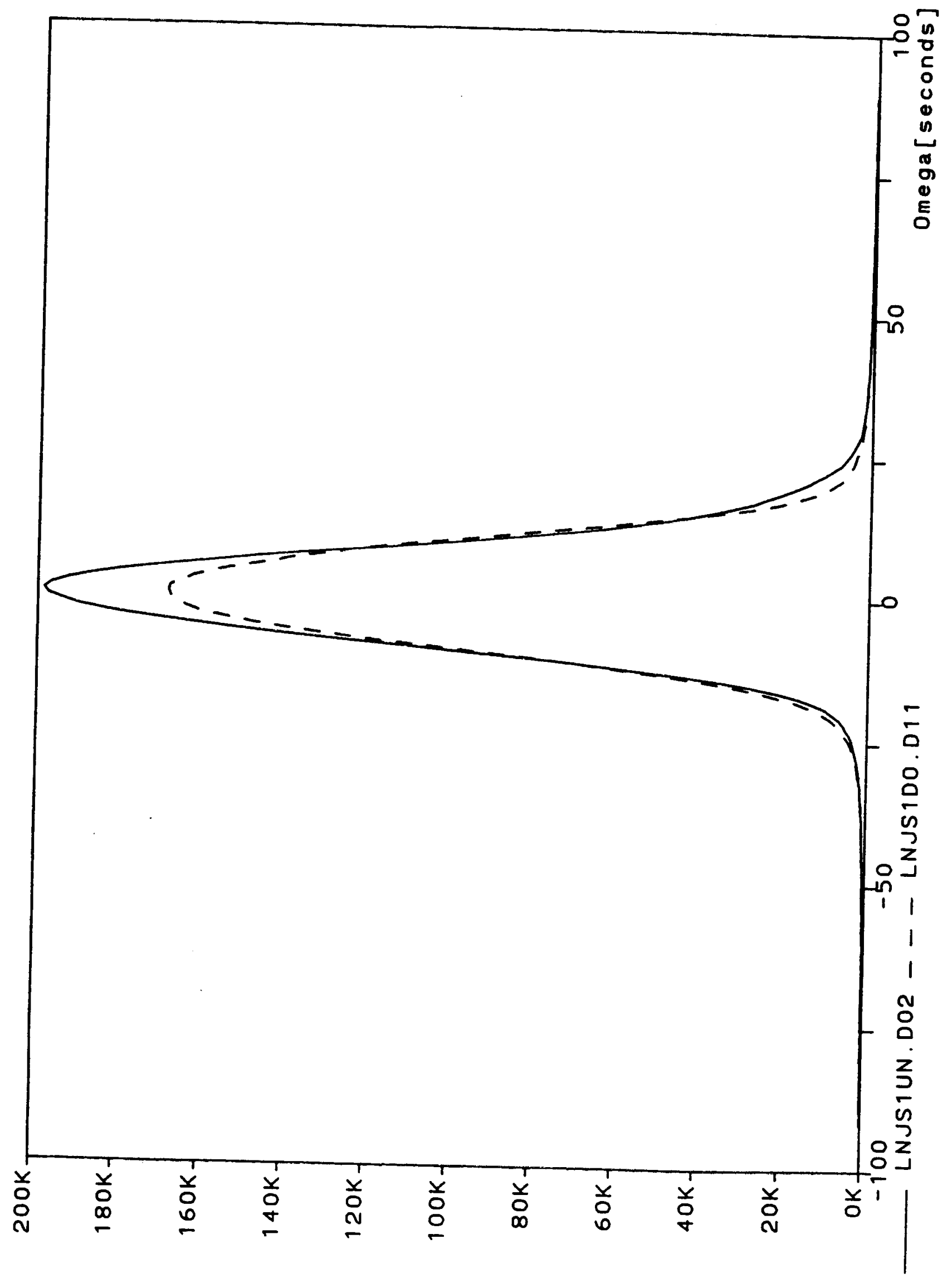



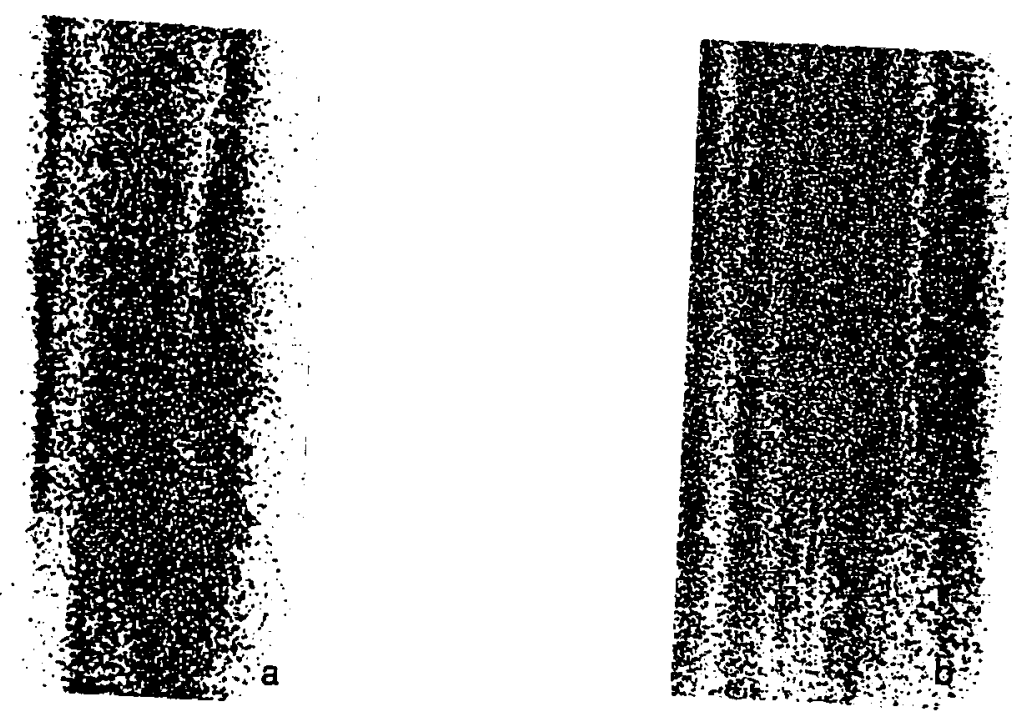

Fig. 6 


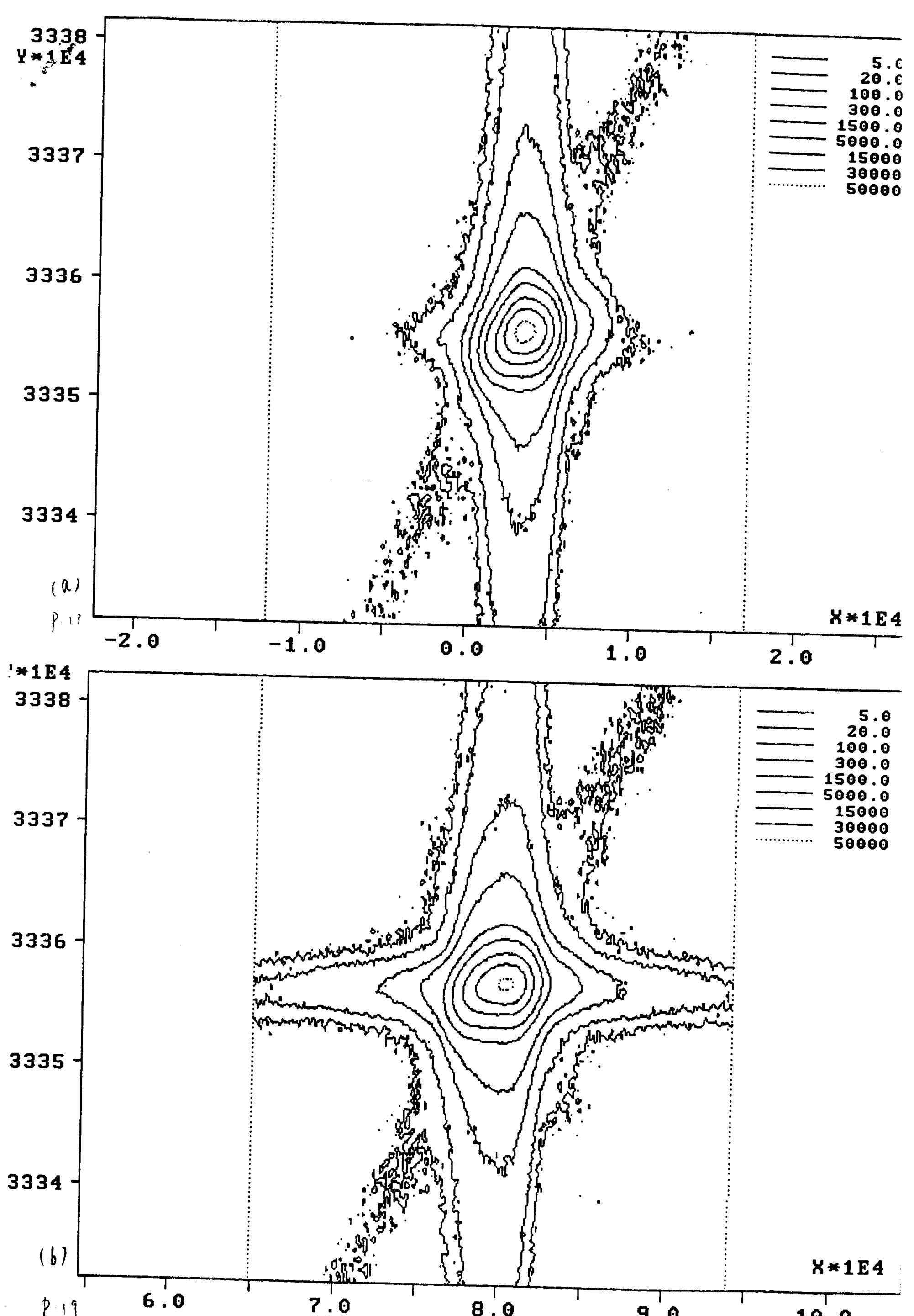



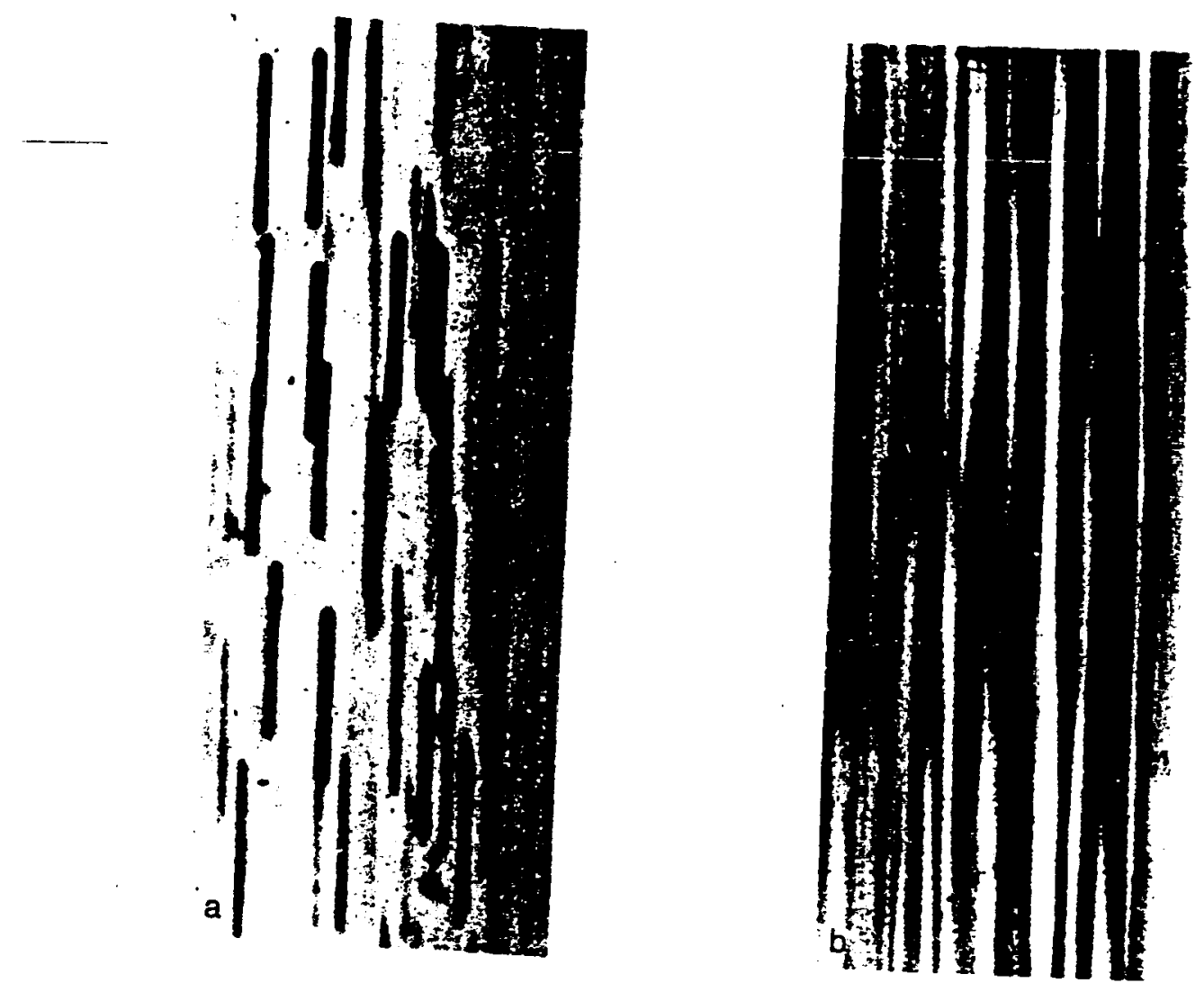

Fig. 8 